\title{
PARAMETRIZED LEGENDRE AND LAGRANGE VARIETIES
}

\author{
By Goo IsHIKAWA
}

\section{Introduction}

Legendre varieties and Lagrange varieties appear in many areas, for instance, geometric optics [A][J2], generalized Cauchy problem for Hamilton-Jacobi equations [G2] [I3], projective geometry [S1], microlocal analysis [P][DP], moduli problem of vector bundles on complex surfaces [Y], symplectic topology [G1] and so on.

In this survey we treat Legendre and Lagrange varieties admitting some parametrizations in complex analytic or $C^{\infty}$ category. Then our study fits with the framework of the theory of singularities of differentiable mappings [AGV][B][D][GWPL][W].

First we introduce the notion of a "front hypersurface" by the property that the Nash modification projects to the hypersurface itself finitely to one. The Nash modification, in this case, is the closure of the lifting of the regular points set to the projective cotangent bundle of the manifold where the hypersurface lies in: The projective cotangent bundle is identified with the totality of contact elements (tangent hyperplanes) of the base space and it has the natural contact structure [A][S1]. The tangent hyperplanes to the regular points of a front hypersurface form a Legendre submanifold, that is, the maximal dimensional integral submanifold of the contact distribution defined over the projective cotangent bundle and the closure of this natural lifting might be regarded as a Legendre variety. In fact, a definition of Legendre variety is that it contains an open dense Legendre submanifold. The Legendre variety thus obtained by Nash modification has singularities in general. If the Nash modification is non-singular, then the hypersurface turns out the projection of a Legendre submanifold. Then the front hypersurface is called a wave front set $[\mathrm{A}][\mathrm{Z} 1]$. Remark that, for a generic Legendre submanifold, the projection is finite to one. In the above definition of front hypersurfaces we allow singularities for Nash modification, and to make the definition non-trivial, we add the finiteness condition. (See [LT] for the general theory of limits of tangent spaces.)

We utilize parametrizations of varieties to formulate the notion above mentioned as follows: A mapping $f$ from an $n$-dimensional manifold $N$ to an $n+1$-dimensional manifold $B$ (say, of class $C^{\infty}$ or complex analytic) is called a front mappıng if the set of regular points of $f$ is dense in $N$, and, for each point $x \in N$, the images of the tangent spaces of regular points converge to a tangent hyperplane $T_{x} \subset T_{f(x)} B$ as regular points tend to $x$, and the tangent hyperplanes $\left\{T_{x}\right\}$ depend smoothly or holomorphically on the points $x \in N$. Then we have a $C^{\infty}$ or holomorphic lifting $\tilde{f}$ of $f$ to the projective cotangent bundle $P T^{*} B$. This lifting is an integral mappıng in the sense that the image $\tilde{f}_{*}\left(T_{x} N\right)$ of tangent space to each point $x \in N$ is contained in the contact distribution of

Received May 27, 1993. 
$P T^{*} B$. We call $\tilde{f}$ the Nash lifting of $f$. Under this formulation, if a front mapping $f$ is finite to one, then the image of $\tilde{f}$ projects finitely to one by the projection from $P T^{*} B$ to $B$; this formulation therefore fits to the naive consideration mentioned before.

A front hypersurface or a front mapping appears also as the "graph" of a Lagrange variety $([G 2])$. So we turn our attention to Lagrange varieties. In general, a subset in a symplectic manifold is called a Lagrange varlety if the regular points set is open dense and it is a Lagrange submanifold, that is, the maximal dimensional integral submanifold where the symplectic form vanishes. A type of Lagrange variety in the cotangent bundle $T^{*} X$ of a manifold $X$ is the graph of a closed "multivalued" one form on $M$. The graph of a closed one form on a manifold (in the usual sense) is an example of Lagrange submanifold of the cotangent bundle. Another important example is the conormal bundle of a submanifold: In general the conormal bundles of varieties with singularities form another class of Lagrange varieties in a cotangent bundle. (For the general theory of Lagrangian varieties, see the excellent survey [G2].)

We shall study also Lagrange varieties through parametrizations of them. (For studies from the viewpoint of "generating families", see [Z2][ZR][J1][JZ].) A $C^{\infty}$ or holomorphic mapping $f$ from an $n$-dimensional manifold $N$ to a $2 n$-dimensional symplectic manifold $(M, \omega)$ with a symplectic form $\omega$ is called an ssotropıc mappıng if the pullback $f^{*} \omega$ is the zero form. In other word, an isotropic mapping is a parametrization of "integral variety" of the differential equation $\omega=0$ on $M$. (For the general theory of symplectic manifolds, see $[\mathrm{AM}][\mathrm{W}]$, for instance.)

If $M$ is the cotangent bundle $T^{*} X$ over a manifold $X$ and the symplectic form $\omega$ is the exterior differential $d \alpha$ of the canonical (Louville) form $\alpha$, then, by Poincaré's Lemma, there exists a function $e$ locally in $N$ such that the exterior differential $d e$ is equal to the pullback $f^{*} \alpha$. We call $e$ a generatıng function of $f$. Remark that, if the singular locus of $\pi \circ f$ is nowhere dense, where $\pi$ is the projection from $T^{*} X$ to $X$, then a isotropic lifting $f$ is uniquely determined from its generating function. The graph of $f$ is the front mapping $(\pi \circ f, e)$ from $N$ to $X \times \mathbf{C}$. Notice that the Nash lifting of $(\pi \circ f, e)$ is equal to the integral mapping $(f, e)$ from $N$ to $T^{*} X \times \mathbf{C}$, which is identified with an affine part of $P T^{*}(X \times \mathbf{C})$.

The classification of front mappings (in the sense of [DP]), induces the Lagrange classification of isotropic mappings through their graphs: Two isotropic map-germs $f$ and $f^{\prime}$ are Lagrange equivalent if and only if their graphs $(f, e)$ and $\left(f^{\prime}, e^{\prime}\right)$ are strict right-left equivalent and it is also equivalent to their Nash liftings are strict Legendre equivalent. Here the word "strict" means that the diffeomorphism of $X \times \mathbf{C}$ in the usual definition of the equivalences should be an isomorphism of the additive $\mathbf{C}$ line bundre $X \times \mathbf{C}$ over $X$.

In recent work [I4][I5][I6], we classify front mappings and therefore Legendre and Lagrange varieties. (See also [I2][I3].) Then we always encounter a certain module describing the ramification of a finite mapping. We also utilize this module to classify differential equations [HIIY]. Let $f$ be a germ of front mapping from $N, x$ to $B$ Then there exists a coordinate $\left(y_{1}, \ldots, y_{n+1}\right)$ around $(B, f(x))$ such that the tangent hyperplane $T_{x}$ is defined by $d y_{n+1}=0$ in $T_{f(x)} B$. If we write $f=\left(g_{1}, \ldots, g_{n}, h\right)$ under this coordinate, then, since $f$ is a front map-germ, we see that $d h=a_{1} d g_{1}+\cdots+a_{n} d g_{n}$, for some $C^{\infty}$ or holomorphic function-germs $a_{1}, \ldots, a_{n}$ on $N, x$. We are thus led to consider the module of all functions $h$ such that the exterior derivative of $h$ is a functional linear 
combination of the exterior derivatives of $g_{1}, \ldots, g_{n}$. We call this module the ramification module of the map-germ $g=\left(g_{1}, \ldots, g_{n}\right)$.

We study on the "finiteness" of the ramification module in $\S 1$.

A classification of front mappings, Legendre varieties and Lagrange varieties under some restrictions are given in $\S 2$. (For the detailed proof, see [I6]).

Another important example of front hypersurfaces is the developable of a curve in an affine space or in an projective space: The ruled surface by the tangent lines to a space curve is called the tangent developable surface of the curve. In general the developable of a curve in $(n+1)$-dimensional projective space is defined as the hypersurface "ruled" by osculating $(n-1)$-subspaces to the curve. These singular hypersurfaces appear also in singular solutions of homogeneous Monge-Ampère equations and give examples for "isotropic" deformations of plane curve singularities. The developable are in some sense quite special front varieties in general. However we achieve the classification of developables for rather general cases: We give the local classification of developables in the complex category in §3. (For the similar result in $C^{\infty}$ category, see [I4][I5].)

Hereafter all mappings, diffeomorphisms and vector fields are assumed of class $C^{\infty}$ or holomorphic according to the context.

This paper is written under the stimulation of the Hanoi workshop: I would like to thank the organizers for the nice hospitality. I would like to thank also Professors D. Mond, S. Izumiya and J. Steenbrink for helpful information.

\section{Ramification module}

Let $X$ be a germ of complex analytic space and $g=\left(g_{1}, \ldots, g_{p}\right): X \longrightarrow \mathbf{C}^{p}$ be a finite holomorphic map-germ. Let $\Omega_{X}^{2}$ denote the germ of sheaf of holomorphic differential $i$-forms on $X$. Then $\Omega=\Omega_{X}=\sum_{\imath} \Omega_{X}^{2}$ is a graded differential $\mathcal{O}_{X}$-algebra with the differential $d$. Consider the graded differential ideal $I$ generated by $d g_{1}, \ldots, d g_{p}$ in $\Omega_{X}$. Then $d: \Omega / I \longrightarrow \Omega / I$ is a $g^{*} \mathcal{O}_{p}$-homomorphism. In fact, for a form $\omega$ on $X$ and a function $a$ on $\mathbf{C}^{p}$, we have

$$
d(a \circ g \cdot \omega)=\left(\sum_{J} \frac{\partial a}{\partial y_{j}} \circ g d g_{j}\right) \omega+a \circ g d \omega \equiv a \circ g d \omega, \quad \bmod . I .
$$

Moreover $d: g_{*}(\Omega / I) \longrightarrow g_{*}(\Omega / I)$ is an $\mathcal{O}_{p}$-homomorphism.

We then set $\mathcal{R}_{g}=\mathcal{H}^{0}\left(g_{*}(\Omega / I), d\right)=\operatorname{Ker}\left(d: g_{*} \mathcal{O}_{X} \longrightarrow g_{*}(\Omega / I)^{1}\right)$. Remark that $\mathcal{O}_{p} \subset \mathcal{R}_{g} \subset g_{*} \mathcal{O}_{X}$. We further set $\mathcal{R}_{g}^{-}=\mathcal{R}_{g} / \mathcal{O}_{p}$. We call $\mathcal{R}_{g}$ (resp. $\mathcal{R}_{g}^{-}$) the ramification module (resp. reduced ramification module) of $g$. We denote $R_{g}$ (resp. $R_{g}^{-}$) the corresponding stalk at the base point of the germ $X$. (The notation $R_{g}$ is borrowed from [M'2]. In [I3][I4][I5], we used the notation $H_{g}$ instead. Similar modules are also considered in [S][M2][Z2] from various motivations.)

Proposition 1.1. $\mathcal{R}_{g}$ (resp. $\mathcal{R}_{g}^{-}$) is a coherent $\mathcal{O}_{p}$-module.

Proof. Since $g_{*}(\Omega / I)$ is $\mathcal{O}_{p}$-coherent, the cohomology $\mathcal{H}=\operatorname{Ker} d / \operatorname{Im} d$ of the complex $\left(g_{*}(\Omega / I), d\right)$ is also $\mathcal{O}_{p}$-coherent.

CoRollary 1.2. If $1, h_{1}, ., h_{r}$ generate $R_{g}$ as $\mathcal{O}_{p}$-module, then $\left(g ; h_{1}, \ldots, h_{r}\right)$ : 
$X \longrightarrow \mathbf{C}^{p} \times \mathbf{C}^{r}$ is an injectıve map-germ. (cf. [M'2][I1][12]). Then

Proof. Since $\mathcal{R}_{g}$ is coherent, $1, h_{1}, \ldots, h_{r}$ generate $\mathcal{R}_{g}$ locally. Take $y \in \mathbf{C}^{p}$ near 0.

$$
\mathcal{R}_{g, y}=\left\{k \in \mathcal{O}_{g^{-1}(y)} \mid d k \in \sum_{i=1}^{p} \mathcal{O}_{g^{-1}(y)} d g_{i}\left(\subset \Omega_{g^{-1}(y)}^{1}\right)\right\}
$$

contains $g_{*} \mathbf{C}_{X}$, where $\mathbf{C}_{X}$ is the constant sheaf. So $h_{1}, \ldots, h_{r}$ separate the points of $g^{-1}(y)$.

Next we turn to the $C^{\infty}$ case. Let $g: \mathbf{R}^{n}, 0 \longrightarrow \mathbf{R}^{p}, 0$ be a finite $C^{\infty}$ map-germ. (The ring $E_{n}$ of $C^{\infty}$ function-germs on $\mathbf{R}^{n}, 0$ is finite over $E_{p}$ via $g^{*}$.) We set $\mathrm{kr} g=$ $\operatorname{dim} \operatorname{Ker} T_{0} g$. To show $R_{g}=\left\{h \in E_{n} \mid d h \in \sum_{i=1}^{p} E_{n} d g_{i}\right\}$ is a finite $E_{p}$-module is unsolved yet in $C^{\infty}$ case. But in particular case $\mathrm{kr} g \leqq 1$, we have

THEOREM 1.3. Let $g: \mathbf{R}^{n}, 0 \longrightarrow \mathbf{R}^{p}, 0$ be a finıte $C^{\infty}$ map-germ. If $k r g \leqq 1$, then $R_{g}$ is a finitely generated $E_{p}$-module via $g^{*}$. Therefore $S \subset R_{g}$ generates $R_{g}$ over $E_{p}$ if and only if $S$ generates $R_{g} / m_{p} R_{g}$ over $\mathbf{R}$.

To prove Theorem 1.3, we link to the unfolding theory of map-germs [GWPL] and we will recover a new aspect of the unfolding theory.

An unfolding of a map-germ $g$ is a triple $(G ; i, j)$ of a map-germ $G: \mathbf{R}^{N}, 0 \longrightarrow \mathbf{R}^{P}, 0$, and immersions $i: \mathbf{R}^{n}, 0 \longrightarrow \mathbf{R}^{N}, 0$ and $j: \mathbf{R}^{p}, 0 \longrightarrow \mathbf{R}^{P}, 0$ such that the following is a fiber square:

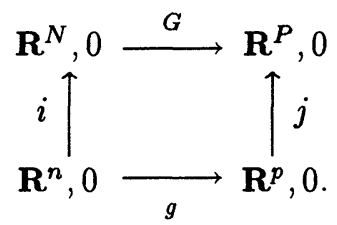

LeMmA 1.4. Let $(G ; i, j)$ be an unfolding of $g$. Then $i^{*}: E_{N} \longrightarrow E_{n}$ induces $i^{*}: R_{G} \longrightarrow R_{g}$ which is a homomorphısm over $j^{*}: E_{P} \longrightarrow E_{p}$, that $\imath s, i^{*}\left\{\left(G^{*} a\right) h\right\}=$ $g^{*}\left(j^{*} a\right) i^{*} h, a \in E_{P}, h \in R_{G}$.

Proof. Let $h \in R_{G}$. Then $d(h \circ i)=\left(\sum a_{\ell} d G_{\ell}\right) \circ i=\sum a_{\ell} \circ i d\left(G_{\ell} \circ i\right)=\sum a_{\ell} \circ i d\left(j_{\ell} \circ g\right)$. Since each $j_{\ell} \circ g \in R_{g}$, we have $d(h \circ i)$ belongs to $\sum E_{n} d g_{\ell}$. Thus $h \circ i \in R_{g}$.

We call $(G ; i, j)$ an admissible unfolding if $i^{*}: R_{G} \longrightarrow R_{g}$ is surjective.

The following is easy to verify:

LeMma 1.5. If $(G ; i, j)$ is admissible, and $R_{G}$ is finite over $E_{P}$, then $R_{g}$ is fintte over $E_{p}$.

Proposition 1.6. If $k r g \leqq 1$, then any unfolding of $g$ is admissible. 
Proof. Let $(G ; i, j)$ be an unfolding of $g$. Then, for some coordinates, $G: \mathbf{R}^{n} \times$ $\mathbf{R}^{m}, 0 \longrightarrow \mathbf{R}^{p} \times \mathbf{R}^{m}, 0$ with $G(x, \lambda)=\left(G_{\lambda}(x), \lambda\right), i(x)=(x, 0)$ and $j(y)=(y, 0)$. Further, $g(x)=\left(x^{\prime}, \phi\left(x^{\prime}, t\right)\right)$ and $G_{\lambda}(x)=\left(x^{\prime}, \phi_{\lambda}\left(x^{\prime}, t\right)\right)$, where $x^{\prime}=\left(x_{1}, \ldots, x_{n-1}\right)$ and $t=x_{n}$. Remark that $h \in R_{g}$ if and only if $\partial h / \partial t=a \partial \phi / \partial t$ for some $a \in E_{n}$. Define $\tilde{h} \in E_{n+m}$, by

$$
\tilde{h}=\int_{0}^{t} a \partial \phi_{\lambda} / \partial t d t+h\left(x^{\prime}, 0\right) .
$$

Then $\tilde{h} \in R_{G}$ and

$$
i^{*} \tilde{h}=\int_{0}^{t} a \partial \phi / \partial t d t+h\left(x^{\prime}, 0\right)=h .
$$

Example 1.7. Let $g=\left(x_{1}^{2}, x_{2}^{2}\right)$ and $G=\left(x_{1}^{2}+\lambda x_{2}, x_{2}^{2}, \lambda\right)$. Then the restriction $R_{G} \longrightarrow R_{g}$ is not surjective. So the unfolding $G$ of $g$ is not admissible.

Remark 1.8. If an unfolding $G^{\prime}: \mathbf{R}^{N}, 0 \longrightarrow \mathbf{R}^{P}, 0$ of $g$ is equivalent ([GWPL]) to $G$, then $R_{G^{\prime}}$ is isomorphic to $R_{G}$ over a ring isomorphism of $E_{P}$.

Proof of Theorem 1.3. By Lemma 1.5, Proposition 1.6 and Remark 1.8, it suffices to check for the stable mapping of type $A_{k}: g=\left(x_{1}, \ldots, x_{n-1}, t^{k+1}+x_{1} t^{k-1}+\cdots+x_{k-1} t\right)$. Then this fact is proved in [I1][I2][I3] already.

Corollary 1.9. Let $g: \mathbf{R}^{n}, 0 \longrightarrow \mathbf{R}^{n}, 0$ (resp. $g: \mathbf{C}^{n}, 0 \longrightarrow \mathbf{C}^{n}, 0$ ) be a finite $C^{\infty}$ (resp. holomorphic) map-germ of form $g\left(x^{\prime}, t\right)=\left(x^{\prime}, u\left(x^{\prime}, t\right)\right)$ of multiplicity $k+1$, where $x^{\prime}=\left(x_{1}, \ldots, x_{n-1}\right)$. Then a subset $S$ of $R_{g}$ generates $R_{g}$ over $E_{n}$ (resp. $\left.\mathcal{O}_{n}\right)$ via $g^{*}$ if and only if the set $\left\{\right.$ ord $\left._{t} h(0, t) \mid h \in S\right\}$ contains $\{0, k+2, \ldots, 2 k+1\}$.

\section{Classification of generic front hypersurfaces}

Let $f: N^{n} \longrightarrow B^{n+1}$ be a $C^{\infty}$ front mapping. Then there exists a unique integral lifting $\tilde{f}: N \longrightarrow P T^{*} B$ (the Nash lifting of $f$ ). Thus we have an injective mapping $\mathcal{N}: C_{F R}^{\infty}(N, B) \longrightarrow C_{I}^{\infty}\left(N, P T^{*} B\right), \mathcal{N}(f)=\tilde{f}$, where $C_{F R}^{\infty}(N, B)$ is the set of $C^{\infty}$ front mappings from $N$ to $B$ and $C_{I}^{\infty}\left(N, P T^{*} B\right)$, is the set of $C^{\infty}$ integral mappings from $N$ to $P T^{*} B$. We give the induced topology on $C_{F R}^{\infty}(N, B)$ from the Whitney $C^{\infty}$ topology of $C_{I}^{\infty}\left(N, P T^{*} B\right) \subset C^{\infty}\left(N, P T^{*} B\right)$.

We will give a generic classification of front mappings of kernel rank at most one in the $C^{\infty}$ case. We set

$$
C_{F R}^{\infty}(N, B)^{1}=\left\{f \in C_{F R}^{\infty}(N, B) \mid \operatorname{kr} f_{x} \leqq 1, \text { for any } x \in N\right\} .
$$

Let $f \in C_{F R}^{\infty}(N, B)^{1}$. We shall impose some genericity conditions to $f$. Then $f$ is reduced to some normal form. (For this point, the reduction is valid also in holomorphic case).

First, under a genericity condition, for any $x \in N$, the germ $f_{x}$ is right-left equivalent to $\left(g\left(x^{\prime}, t\right), h\left(x^{\prime}, t\right)\right): \mathbf{R}^{n}, 0 \longrightarrow \mathbf{R}^{n} \times \mathbf{R}, 0$ with $g\left(x^{\prime}, t\right)=\left(x^{\prime}, u\left(x^{\prime}, t\right)\right)$ where $x^{\prime}=$ $\left(x_{1}, ., x_{n-1}\right)$ and $u=e(k+1)+x_{1} e(k-1)+\cdots+x_{k-1} e(1), \partial h / \partial t=w \partial u / \partial t$ for some $w \in E_{x^{\prime}, t}, 0 \leqq k \leqq n$. Here we set $e(i)=t^{i} / i$ !. Then we see $h \in R_{g}$. (See $\S 1$.) Then $w$ is 
uniquely determined from $h$. By Corollary 1.9 , we have

$$
h=A_{0} \circ g+\sum_{j=1}^{k} A_{\jmath} \circ g u_{\jmath}, \quad \text { where } \quad u_{\jmath}=\int_{0}^{t} e(j) \frac{\partial u}{\partial t} d t .
$$

Remark that $\operatorname{ord}_{t} u_{j}(0, t)=k+j+1,(1 \leqq j \leqq k)$. Then we see

$$
w=\left(\partial A_{0} / \partial q_{n}\right) \circ g+\sum_{\jmath}\left(\partial A_{\jmath} / \partial q_{n}\right) \circ g u_{j}+\sum_{\jmath} A_{\jmath} \circ g e(j) .
$$

Thus, for $1 \leqq j \leqq k, \partial^{\jmath} w / \partial t^{j} \equiv A, \circ g, \bmod .\left\langle x_{1}, \ldots, x_{k-1}, t\right\rangle \subset E_{n}$. Next we impose the generic condition on $(u, w)$ : For some $\ell, 0 \leqq \ell \leqq n-k$,

$$
\partial w / \partial t(0)=\cdots=\partial^{\ell} w / \partial t^{\ell}(0)=0, \partial^{\ell+1} w / \partial t^{\ell+1}(0) \neq 0
$$

and that

$$
\left(\partial u / \partial t, \ldots, \partial^{k} u / \partial t^{k} ; \partial w / \partial t, \ldots, \partial^{\ell} w / \partial t^{\ell}\right)
$$

is a submersion.

Now we introduce the local models $f_{n, k, \ell}: \mathbf{R}^{n}, 0 \longrightarrow \mathbf{R}^{n+1}, 0$ by $f_{n, k, \ell}\left(x^{\prime}, t\right)=$ $\left(x^{\prime}, u, h\right)$,

$$
u=e(k+1)+x_{1} e(k-1)+\cdots+x_{k-1} e(1), \quad \text { and } \quad h=u_{\ell+1}+\sum_{i=k}^{k+\ell-1} x_{i} u_{k+\ell-\imath} .
$$

THEOREM 2.1. There exists an open dense subset $\mathcal{G} \subset C_{F R}^{\infty}(N, B)^{1}$ such that, for any $f \in \mathcal{G}$ and for any $x \in N$, the germ $f_{x}: N, x \longrightarrow B$ is $C^{\infty}$ right-left equivalent to $f_{n, k, \ell}, 0 \leqq \ell \leqq k \leqq n, \ell+k \leqq n$.

Remark 2.2. The multiplicity of $f_{n, k, \ell}$ is equal to $k+1$ and the multiplicity of the Nash lifting $\tilde{f}_{n, k, \ell}$ is equal to $\ell+1$. So the local models are inequivalent to each other. We call the singularity $f_{n, k, \ell}$ of front mapping of type $A_{k+1, \ell}$, when $n$ is fixed. Then the $A_{k+1,0}$ singularities are just the usual $A_{k+1}$ singularities of wave front sets [AGV].

CoRollary .2.3. For a generic integral mapping $F: N \longrightarrow P T^{*} B$ with the kernel rank $\pi \circ F \leqq 1$, and for any $x \in N, F_{x}$ is Legendre equivalent to one of $\tilde{f}_{n, k, \ell}, 0 \leqq \ell \leqq$ $k \leqq n, \ell+k \leqq n$.

Outline of the proof of Theorem 2.1. If $k \leqq \ell$, then $f_{x}$ is equivalent to $f_{n, k, k}$ as proved in [I3]. If $\ell<k$, then $f_{x}$ is equivalent in this case to $(g, h)$ with

$$
h=x_{k} u_{\ell}+x_{k+1} u_{\ell+1}+\ldots+x_{k+\ell-1} u_{1}+\alpha,
$$

for some $\alpha \in R_{g}$ with $\partial \alpha / \partial t \in t^{\ell+1} E_{x^{\prime}, t} \partial u / \partial t$. Write $\alpha=u_{\ell+1}+\beta$. Set, for $s \in \mathbf{R}$,

$$
h_{s}=x_{k} u_{\ell}+\cdots+x_{k+\ell-1} u_{1}+u_{\ell+1}+s \beta \text {. }
$$

Then we claim that the family of front map-germs $\left(g, h_{s}\right)$ is trivial. To see this, write

$$
t^{2} \partial u / \partial t=\sum_{\jmath=1}^{k-1} a_{i j} \circ g \partial u / \partial x_{\jmath}+b_{i} \circ g \partial u / \partial t+c \circ g
$$


for some $a_{i j}, b_{i}, c \in m_{y}$. Then set

$$
X_{\imath}=\left(t^{i}-b_{i} \circ g\right) \partial / \partial t-\sum_{j=1}^{k-1} a_{i j} \circ g \partial / \partial x_{\jmath},
$$

which is $g$-lowerable: $g_{*} X_{\imath}=g^{*} Y_{\jmath}, Y_{\imath}=\sum_{j=1}^{k-1} a_{i j} \partial / \partial y_{j}+c \partial / \partial y_{n}$, with $X_{i}(0)=$ $0, Y_{i}(0)=0$. Moreover the $k+1$ elements

$$
1, \frac{\partial h_{s}}{\partial x_{k}}, \ldots, \frac{\partial h_{s}}{\partial x_{k+\ell-1}}, X_{1} h_{s}, \ldots, X_{k-\ell} h_{s}
$$

generate $R_{g}$ over $g^{*} E_{y}$ by Corollary 1.9 . Therefore $\beta=X h_{s}+a_{0} \circ g$, where

$$
X=\sum_{j=1}^{\ell} a_{\jmath} \circ g \partial / \partial x_{j}+\sum_{\rho=1}^{k-\ell} b_{\rho} \circ g X_{\rho}
$$

for some $a_{j}, b_{\rho} \in m_{y}, 0 \leqq j \leqq \ell, 1 \leqq \rho \leqq k-\ell$. Thus the triviality is shown similarly as in [I5]. Therefore $f_{x}=\left(g, h_{1}\right)$ is equivalent to $f_{n, k, \ell}=\left(g, h_{0}\right)$ as required.

Remark 2.4. To trivialize the family $\left(g, h_{s}\right)$, we need only additive $\mathbf{R}$ line bundle isomorphisms $\mathbf{R}^{n} \times \mathbf{R} \longrightarrow \mathbf{R}^{n},\left(y_{1}, \ldots, y_{n}, y_{n+1}\right) \mapsto\left(y_{1}, \ldots, y_{n}\right)$. We then obtain a result on the classification of isotropic mappings through their graphs.

Corollary 2.5. For a generic isotropıc mapping $f: N \longrightarrow T^{*} X$ with $k r \pi \circ f \leqq$ 1 , the Lagrange equivalence classes of $f_{x},(x \in X)$, are determined by the multiplicities of $f_{x}$ and $\pi \circ f_{x}$. (cf. [I3]).

Remark 2.6. The transversality theorem holds for integral mappings to a contact manifold and for isotropic mappings to a symplectic manifold of kernel rank at most one. Then we have the generic Legendre classification of integral mappings $F: N \longrightarrow P T^{*} B$ of kernel rank at most one for $n=\operatorname{dim} N \leqq 3$ :

$$
\begin{aligned}
& n=1: A_{1}, A_{2}, \\
& n=2: \text { Moreover } A_{3}, A_{2,1}, \\
& n=3: \text { Moreover } A_{4}, A_{3,1}, D_{4} .
\end{aligned}
$$

\section{Singularities of developables}

In [I4][I5], we study the local $C^{\infty}$ classification of singularities appearing in developables. This result unifies and generalizes the previous results of Cleave, Gaffney, du Plessis, Arnol'd, Shcherbak and Mond. (See [M'1][S1][S2].)

Here we state the similar result in complex analytic category: Consider a holomorphic curve $\gamma: M \longrightarrow \mathbf{C} P^{n+1}$, where $M$ is a one-dimensional complex manifold and $n \geqq 1$. We call the germ $\gamma_{p}$ at a point $p \in M$ of finite osculation-type (or simply, of finite type) $\mathbf{A}=\left(a_{1}, a_{2}, \ldots, a_{n+1}\right)$ if there exist a holomorphic coordinate $t$ of $(M, p)$ and an affine coordinate $\left(x_{1}, \ldots, x_{n+1}\right)$ of $\mathbf{C} P^{n+1}$ centered at $\gamma(p)$ such that $\gamma$ is represented by

$$
x_{1}=t^{a_{1}}+o\left(t^{a_{1}}\right), \quad \ldots \quad x_{n+1}=t^{a_{n+1}}+o\left(t^{a_{n+1}}\right),
$$

where each $a_{i}$ is a natural number and $1 \leqq a_{1}<\cdots<a_{n+1}$. Then we write type $\left(\gamma_{p}\right)=\mathbf{A}$.

For each $p \in M$ where $\gamma_{p}$ is of finite type and for each $i,(0 \leqq i \leqq n+1)$, there exists the most osculating linear subspace to $\gamma$ at $p$ in $T_{\gamma(p)} \mathbf{C} P^{n+1}$ of dimension $i$. We call it the 
osculating $i$-subspace and denote by $O_{i}(\gamma, p)$. This subspace is identified with $\left\{x_{i+1}=\right.$ $\left.\cdots=x_{n}=0\right\}$ under the above affine representation of $\gamma_{p}$. The corresponding projective subspace of $\mathbf{C} P^{n+1}$ through $\gamma(p)$ of dimension $i$ is also denoted by $O_{i}(\gamma, p)$. The type of a curve describes the order of tangency to each osculating subspace, and it is the simplest local projective invariant of the curve. Further we define the osculating $i$-bundle $O_{i}(\gamma)=$ $\bigcup_{p \in M} O_{i}(\gamma, p)$ in the pullback bundle $\gamma^{-1} T \mathbf{C} P^{n+1}$. The natural parametrization $\operatorname{dev}(\gamma)$ : $O_{n-1}(\gamma) \longrightarrow \mathbf{C} P^{n+1}$ defined by $(p, q) \mapsto q$, where $q \in O_{n-1}(\gamma, p)\left(\subset \mathbf{C} P^{n+1}\right)$, is called also a developable of $\gamma$.

The developable of $\gamma$ is a front mapping: The Nash lifting of $\operatorname{dev}(\gamma)$ to $P T^{*} \mathbf{C} P^{n+1}$ is in fact the projective conormal bundle of the dual curve $\gamma^{*}: M \longrightarrow \mathbf{C} P^{n+1^{*}}$ in the dual projective space $\mathbf{C} P^{n+1^{*}}$ defined by $\gamma^{*}(p)=O_{n}(\gamma, p)$ through the identification $P T^{*} \mathbf{C} P^{n+1} \cong P T^{*} \mathbf{C} P^{n+1 *}$.

The germ $\operatorname{dev}(\gamma)_{p}$ of $\operatorname{dev}(\gamma)$ at $(p, 0)$ is determined up to the projective equivalence by the projective class of $\gamma_{p}$. Instead, we consider a weaker equivalence, that is, holomorphic right-left equivalence: $\operatorname{dev}(\gamma)_{p}$ is holomorphically equivalent to $\operatorname{dev}\left(\gamma^{\prime}\right)_{p^{\prime}}$ if there exist holomorphic diffeomorphisms $\sigma: O_{n-1}(\gamma),(p, 0) \longrightarrow O_{n-1}\left(\gamma^{\prime}\right),(p, 0)$ and $\tau: \mathbf{C} P^{n+1}, \gamma(p) \longrightarrow \mathbf{C} P^{n+1}, \gamma(p)$ such that $\tau \circ \operatorname{dev}(\gamma)_{p}=\operatorname{dev}\left(\gamma^{\prime}\right)_{p^{\prime}} \circ \sigma$. Then it is natural to ask whether or not a type of a curve-germ $\gamma_{p}$ determines the holomorphic right-left equivalence class of map-germ $\operatorname{dev}(\gamma)_{p}: \mathbf{A}$ type $\mathbf{A}$ of a curve-germ is called determinatıve if type $\left(\gamma_{p}\right)=\operatorname{type}\left(\gamma_{p^{\prime}}^{\prime}\right)$ implies that $\operatorname{dev}(\gamma)_{p}$ is equivalent to $\operatorname{dev}\left(\gamma^{\prime}\right)_{p^{\prime}}$.

The following result gives the complete characterization of determinative types:

Theorem 3.1. A type $\mathbf{A}$ of a holomorphic curve-germ in $\mathbf{C} P^{n+1}$ is determinative if and only if $\mathbf{A}$ is one of following types:

$(\mathrm{I})_{n, r} \mathbf{A}=(1,2, \ldots, n, n+r), \quad r=1,2, \ldots$,

$(\text { II })_{n, i} \quad \mathbf{A}=(1,2, \ldots, i, i+2, \ldots, n+1, n+2), \quad 0 \leqq i \leqq n-1$,

(III) ${ }_{n} \mathbf{A}=(3,4, \ldots, n+2, n+3)$,

$(\mathrm{IV})_{m} \mathbf{A}=(2,2 m+1), m=2,3, \ldots$,

(V) $\mathbf{A}=(3,5)$,

(VI) $\mathbf{A}=(1,3,5)$

Further, in this case, for any $\gamma_{p}$ of type $\mathbf{A}$, the map-germ dev $(\gamma)_{p}$ is holomorphically right-left equivalent to $\left(x^{\prime}, U\left(x^{\prime}, t\right), U_{r}\left(x^{\prime}, t\right)\right): \mathbf{C}^{n}, 0 \longrightarrow \mathbf{C}^{n+1}, 0$, where $\left(x^{\prime}, t\right)=$ $\left(x_{1}, \ldots, x_{n-1}, t\right)$ is a coordinate of $\left(\mathbf{C}^{n}, 0\right)$,

$$
U\left(x^{\prime}, t\right)=\frac{t^{a_{n}}}{a_{n} !}+x_{1} \frac{t^{a_{n}-a_{1}}}{\left(a_{n}-a_{1}\right) !}+\cdots+x_{n-1} \frac{t^{a_{n}-a_{n-1}}}{\left(a_{n}-a_{n-1}\right) !},
$$

$r=a_{n+1}-a_{n}$ and

$$
U_{r}\left(x^{\prime}, t\right)=\int_{0}^{t} \frac{t^{r}}{r !} \frac{\partial U}{\partial t} d t
$$

Remark 3.2. For plane curves, that $\mathbf{A}=\left(a_{1}, a_{2}\right)$ is determinative means that $\operatorname{type}\left(\gamma_{p}\right)=\operatorname{type}\left(\gamma_{p^{\prime}}^{\prime}\right)$ implies the curve-germs $(\gamma)_{p},\left(\gamma^{\prime}\right)_{p^{\prime}}$ themselves are holomorphically equivalent, and the determinative types are $(1,1+r), r=1,2, \ldots,(2,3),(3,4),(2,2 m+$ $1), m=2,3, \ldots$ and $(3,5)$. For space curves, the determinative types are

$$
(1,2,2+r), r=1,2, \ldots,(2,3,4),(1,3,4),(3,4,5) \text { and }(1,3,5)
$$

In [S2], it is observed that the developable of a curve of type (IV): $(1,3,5)$ is equivalent to the variety of irregular orbits of the finite reflection group $\mathrm{H}_{3}$ in $\mathbf{C}^{3}$. 
Remark 3.3. We naturally associate Theorem 3.1 with the $A D E$-classification of Dynkin diagrams, finite reflection groups or a simple singularities ([AGV][G']): We do not know however the hidden mathematics behind Theorem 3.1.

Proof of Theorem 3.1. The proof is translated word by word from the proof in the $C^{\infty}$ case [I4][I5] except that we need to use the result in $\S 1$.

\section{REFERENCES}

[AM] R. Abraham, J.E. Marsden, Foundation of Mechanics, 2nd ed., Benjamin, 1978.

[A] V.I. Arnol'd, Singularities of Caustics and Wave Fronts, Kluwer Academic Publishers, 1990.

[AGV] V.I. Arnol'd, S.M. Gusein-Zade, A.N. Varchenko, Singularities of Differentiable Maps I, Birkhäuser, 1985.

[D] J. Damon, The unfolding and determinacy theorems for subgroups of $\mathcal{A}$ and $\mathcal{K}$, Memoirs of Amer. Math. Soc. 50-306 (1984).

[DP] N.H. Du'c, F. Pham, Germs de configurations Legendriennes stables et fonctions d'Airy-Weber généralisées, Ann. Inst. Fourier 41-4 (1991), 905-936.

[G] C.G. Gibson, Singular Points of Smooth Mappings, Pitman, 1979.

[GWPL] C.G. Gibson, K. Wirthmüller, A.A. du Plessis, E.J.N. Looijenga, Topological Stability of Smooth Mappings, Lecture Notes in Math. 552, Springer-Verlag, 1976.

[G'] M. Giusti, Classification des singularités isolées simples d'intersections complètes, Proc. Symp. Pure Math. 40-1, Amer. Math. Soc., 1983, pp. 457-494.

[G1] A.B. Givental', Lagrangian imbeddings of surfaces and unfolded Whitney umbrella, Funkt. Anal. Prilozhen 20-3 (1986), 35-41.

[G2] A.B. Givental', Singular Lagrangian varieties and thier Lagrangian mappings, Itogi Nauki Tekh., Ser. Sovrem. Probl. Mat. (Contemporary Problems of Mathematics) 33, VITINI, 1988, pp. 55-112.

[HIIY] A. Hayakawa, G. Ishikawa, S. Izumiya, K. Yamaguchi, Classification of generic integral diagram and first order ordinary differential equations, to appear, Internat. J. Math.

[I1] G. Ishikawa, Families of functions dominated by distributions of $\mathcal{C}$-classes of mappings, Ann. Inst. Fourier 33-2 (1983), 199-217.

[I2] G. Ishikawa, Parametrization of a singular Lagrangian variety, Trans. Amer. Math. Soc. 331-2 (1992), 787-798.

[13] G. Ishikawa, The local model of an isotropic map-germ arising from one dimensional symplectic reduction, Math. Proc. Camb. Phil. Soc. 111-1 (1992), 103-112.

[14] G. Ishikawa, The determinacy of the envelope of the osculating hyperplanes to a curve, Bull. London Math. Soc. 25 (1993), 603-610.

[I5] G. Ishikawa, Developable of a curve and determinacy relative to osculation-type, to appear, Quart. J. Math.

[I6] G. Ishikawa, Singularities of front hypersurfaces, in preparation.

[J1] S. Janeczko, Generating families for images of Lagrangian submanifolds and open swallowtails, Math. Proc. Camb. Phil. Soc. 100 (1986), 91-107.

[J2] S. Janeczko, Generalized Luneburg canonical varieties and vector fields on quasicaustics, J. Math. Phys. 31-4 (1990), 997-1009.

[JZ] S. Janeczko, H. Zoladek, On singular images of Lagrangian submanifolds, Bull. Polish Acad. Sci. Math. 34 (1986), 215-224.

[LT] D.T. Lê, B. Teissier, Limites d'espaces tangents en géométrie analytique, Comment. Math. Helv. 63 (1988), 540-578. 
[M1] B. Malgrange, Ideals of Differentiable Functions, Oxford Univ. Press, 1966.

[M2] B. Malgrange, Frobenius avec singularités, 2. Le cas general, Invent. Math. 39 (1977), 67-89.

[M'1] D. Mond, Singularities of the tangent developable surface of a space curve, Quart. J. Math. 40-2 (1989), 79-91.

[M'2] D. Mond, Deformations which preserve the non-immersive locus of a map-germ, Math. Scand. 66 (1990), 21-32.

[M] B. Morin, Formes canonique des singularités d'une application différentiable, C.R. Acad. Sc. Paris 260 (1965), 5662-5665.

[P] F. Pham, Singularités Des Systèmes Différentiels De Gauss-Manin, Progress in Math. vol. 2, Birkhäuser, 1979.

[S] K. Saito, On a generalization of de-Rham lemma, Ann. Inst. Fourier 26-2 (1976), 165-170.

[S1] O.P. Scherbak, Projectively dual curves and Legendre singularities, Sel. Math. Sov. 5-4 (1986), 391-421.

[S2] O.P. Scherbak, Wavefront and reflection groups, Russian Math. Surveys 43-3 (1988), $149-194$.

[W] C.T.C. Wall, Finite determinacy of smooth map-germs, Bull London Math. Soc. 13, (1981), 481-539.

[W'] A. Weinstein, Lectures on symplectic manifolds, Regional Conference Series in Math. 29, Amer. Math. Soc., 1977.

[Y] Yun-Gang Ye, Lagrangian subvarieties of the moduli space of stable vector bundles on a regular algebraic surface with $p_{g}>0$, Math. Ann. 295 (1993), 411-425.

[Z1] V.M. Zakalyukin, Reconstructions of fronts and caustics depending on a parameter and versality of mappings, J. of Soviet Math. 27-3 (1984), 2713-2735.

[Z2] V.M. Zakalyukin, Generating ideals of Lagrangian varieties, Theory of Singularities and its Applications, ed. by V.I. Arnol'd, Advances in Soviet Mathematics vol. 1, Amer. Math. Soc., 1990, pp. 201-210.

[ZR] V.M. Zakalyukin, R.M. Roberts, On stable singular Lagrangian varieties, Funct. Anal. Appl. 26-3 (1992), 174-178.

\section{DePaRTMENT OF Mathematics}

HOKKAIDO UNIVERSITY, SAPPORO 060, JAPAN

e-mail: ishikawa@math.hokudai.ac.jp 